\title{
INDICATORI PER LA VALUTAZIONE DELLA POTENZIALITÀ TURISTICO-RICETTIVA DI EDIFICI RURALI TRADIZIONALI
}

\author{
Giovanni Cascone, Simona M.C. Porto
}

\section{INTRODUZIONE}

\section{Introduction}

L'applicazione di un metodo per la valutazione della potenzialità d'uso dei fabbricati rurali tradizionali può costituire una fase molto importante nell'ambito di programmi di sviluppo di aree territoriali omogenee, allorché è necessario scegliere, fra un insieme di alternative possibili, gli edifici sui quali concentrare le risorse economiche disponibili. A tal riguardo, come ormai largamente condiviso dalla comunità scientifica, è opportuno avvalersi di modelli basati sull'analisi multicriteriale infatti, molti benefici (diretti e/o indiretti) prodotti dalle caratteristiche intrinseche degli edifici (cioè, connesse con l'esistenza stessa della risorsa edilizia, indipendentemente da una domanda d'uso) sono, spesso, incommensurabili e/o intangibili e, pertanto, non stimabili attraverso valutazioni economico-monetarie quali, ad esempio le analisi costibenefici [10, 22, 25, 29].

I criteri più frequentemente adottati per la valutazione delle caratteristiche intrinseche dei fabbricati sono: l'importanza storica, l'interesse architettonico, la rilevanza paesaggistica, nonché la potenzialità d'uso. Quest'ultimo criterio, in particolare, esprime la compatibilità di nuove destinazioni d'uso con le caratteristiche tipologiche e morfologiche degli organismi edilizi esistenti $[6,19]$.

Come è noto gli edifici rurali tradizionali costituiscono una chiave di lettura fondamentale per l'inter-

Paper received 22.11.2007; accepted 15.10.2008

Prof. ing. Giovanni Cascone, Ordinario; Dott.ssa ing. Simona M.C. PorTo, Dottore di Ricerca; Dipartimento di Ingegneria Agraria, Sezione Costruzioni e Territorio, Università degli Studi di Catania. La Dott.ssa Simona M.C. Porto ha predisposto la metodologia, svolto le elaborazioni e redatto il testo. Il prof. Giovanni Cascone ha coordinato la ricerca.

The study was partially funded by the C.I.P. INTERREG III B - Archimed 2000-2006; title of the project "Tourism uses of the historic environment. Know-how transfer and quality management practices at community level"; acronym HERODOT. Cod. A.2.054 - 3.2 pretazione del paesaggio rurale $[21,28]$ e, nello stesso tempo, rappresentano una considerevole risorsa edilizia, spesso non utilizzata o sottoutilizzata a causa del mutamento delle condizioni storiche, sociali ed economiche dell'agricoltura [9, 26]. Pertanto, al fine di garantirne la tutela e la salvaguardia, è opportuno che gli interventi di recupero finalizzati al riuso siano definiti in relazione alla valutazione delle loro potenzialità d'uso per la destinazione prefissata.

Come è emerso da precedenti studi $[3,4,5,8]$, i principali fattori che possono influenzare la potenzialità d'uso degli edifici rurali tradizionali per finalità turistico-ricettive sono raggruppabili in due categorie: fattori di contesto territoriale/locale (o estrinseci) e fattori intrinseci.

I fattori di contesto territoriale/locale di un edificio rurale sono:

- le caratteristiche ambientali (paesaggi, boschi, corsi d'acqua, litorali marini, ecc.);

- le caratteristiche culturali (vicinanza di centri storici, monumenti isolati, zone archeologiche, ecc.);

- le caratteristiche dell'attività agricola (indirizzo produttivo, modalità di coltivazione del fondo, trasformazione dei prodotti agricoli);

- le caratteristiche del fondo agricolo (dotazione di servizi, raggiungibilità, possibilità di praticare attività di tempo libero, natura del terreno, forma e dimensioni).

Le caratteristiche elencate possono assumere importanza diversa in relazione al tipo di valutazione da effettuare. Se, ad esempio, il processo valutativo è finalizzato all'erogazione di contributi per la realizzazione di nuove attività agrituristiche, le caratteristiche agricole del fondo dovranno assumere importanza maggiore rispetto ad analoghe valutazioni effettuate per l'esercizio di attività di turismo rurale, Bed and Breakfast, ecc.

I fattori intriseci che possono influenzare la valutazione della potenzialità d'uso turistico-ricettiva di un edificio rurale tradizionale sono: la valenza storica; le caratteristiche architettoniche; i caratteri morfologici; lo stato di conservazione.

Ad oggi, il criterio di valutazione delle istanze per l'assegnazione dei contributi finalizzati al recupero e 
al riuso dei fabbricati rurali favorisce gli edifici e, più in generale, gli insediamenti rurali vincolati ai sensi della Legge n. 1089/39 e s. m. i. per via delle loro caratteristiche di pregio storico e/o architettonico. Generalmente, l'influenza esercitata dai caratteri morfologici sulla possibilità di riuso non rientra tra gli indicatori utilizzati per la scelta dei progetti da finanziare. Ciò costituisce un nodo cruciale per la salvaguardia e la valorizzazione dell'architettura rurale tradizionale poiché, non di rado, gli interventi di recupero attuati trascurano la compatibilità della nuova destinazione funzionale con i caratteri morfologici dei fabbricati rurali da riutilizzare.

In questo lavoro è illustrato un metodo che consente di definire e calcolare indicatori appropriati per la valutazione della potenzialità d'uso turistico-ricettiva di edifici rurali tradizionali.

Al fine di fornire una esemplificazione, il metodo proposto è stato applicato ad un campione di edifici rurali tradizionali ubicati in un'area omogenea della Sicilia sud-orientale particolarmente vocata per attività turistico-ricettive.

\section{METODO PER LA VALUTAZIONE DELLA POTENZIALITÀ D'USO}

\section{Method for the assessment of the potential use}

In questo lavoro viene ripreso ed applicato un metodo definito in precedenti studi $[4,23]$ che consente di definire e calcolare il valore di due di indicatori utili per la valutazione della potenzialità d'uso di edifici rurali tradizionali:

- il grado di adattamento della destinazione d'uso dell'edificio al contesto locale (indicatore n. 1);

- il grado di adattamento della destinazione d'uso ai caratteri morfologici dell'edificio (indicatore n. 2).

La scelta di tali indicatori scaturisce dai contenuti del Decreto Ministeriale 6 ottobre 2005 [13] che ammette la realizzazione di interventi sull'edilizia rurale tradizionale compatibili con le caratteristiche originarie degli edifici e preordinati "alla ricomposizione del rapporto funzionale tra insediamento e spazio produttivo" (art. 2, comma 1, 2).

L'applicazione del metodo proposto nello studio per la valutazione degli indicatori consente di determinare quanto il cambio di destinazione d'uso dei fabbricati rurali tradizionali possa influenzare il mantenimento dei loro caratteri morfologici (indicatore $n$. 1) nonché la qualità del contesto territoriale in cui essi sono inseriti (indicatore n. 2).

Le fasi del metodo sono descritte nei paragrafi che seguono.

\subsection{FASE CONOSCITIVA}

\subsection{Survey}

L'obiettivo della prima fase del metodo è pervenire alla conoscenza dei fattori di contesto territoriali/loca- li ed intrinseci che intervengono nella valutazione della potenzialità d'uso dei fabbricati che compongono il patrimonio edilizio rurale tradizionale da recuperare.

In questa fase del metodo si individua un'area di indagine omogenea sotto il profilo delle caratteristiche territoriali che possono influenzare la possibilità di riuso dei fabbricati rurali tradizionali. A tal proposito, è possibile avvalersi di ricerche di archivio, della cartografia di base nonché degli studi agricolo-forestali e di altri elaborati a supporto degli strumenti urbanistici.

All'individuazione dell'area di indagine segue la messa a punto di schede di rilievo che possono essere articolate secondo schemi proposti per finalità analoghe $[4,8]$. Al fine di ridurre gli oneri necessari allo svolgimento delle campagne di rilievo, è opportuno che i dati raccolti nelle schede siano quelli strettamente necessari a consentire la valutazione della suscettività al riuso.

È opportuno suddividere le informazioni da rilevare in due classi distinte: informazioni acquisibili senza accedere all'interno del fabbricato; informazioni acquisibili accedendo all'interno del fabbricato.

Le prime riguardano principalmente $\mathrm{i}$ fattori di contesto territoriale/locale ed i fattori intrinseci esterni del fabbricato (caratteri formali e funzionali degli elementi di facciata e loro stato di conservazione); le seconde, invece, si riferiscono ai fattori intrinseci interni (distribuzione planimetrica, morfologia e stato di conservazione degli elementi architettonici rilevabili nelle unità funzionali interne all'edificio).

Tale impostazione delle schede consente l'applicazione delle procedure per la valutazione della potenzialità d'uso anche ad edifici inaccessibili. Infatti, nell'ambito di aree omogenee, mediante opportuni studi su idonei campioni [20] (analisi di frequenza, caratterizzazione tipologico - funzionale, ecc.), è possibile ricavare con una certa attendibilità le informazioni anche per gli edifici in cui non è possibile accedere all'interno.

\subsection{FASE VALUTATIVA}

\subsection{Assessment}

Scelta una destinazione funzionale per la quale valutare la potenzialità d'uso di un insieme $m$ di edifici rurali tradizionali, questa fase del metodo prevede per ciascun fabbricato la valutazione dell'indicatore n. 1 e dell'indicatore n. 2.

Poiché alcuni fattori che influenzano la valutazione degli indicatori possono interagire tra loro, è opportuno utilizzare metodi di analisi multicriteriale che consentano di determinare tali interazioni. Tra $\mathrm{i}$ metodi disponibili, è stato scelto un modello già applicato per la valutazione di beni architettonici ed ambientali [25] che consiste nella costruzione di una matrice quadrata $\boldsymbol{X}$ di ordine $n$, le cui $n$ righe ed $n$ colonne si riferiscono agli $n$ fattori da analizzare.

L'elemento $x_{i i}\left(x_{i i} \geq 0\right.$ per $\left.i=1,2, \ldots, n\right)$ della matrice 
$\boldsymbol{X}$ indica il grado di azione attribuito al fattore $\left(x_{i}\right)$ corrispondente alla $i$-esima riga (o $j$-esima colonna). Il grado di azione si assume:

- pari a 1 , se il fattore in esame è presente ad un livello ritenuto normale;

- compreso fra 0 e 1 , se il fattore in esame è presente ad un livello inferiore a quello ritenuto normale;

- maggiore di 1 , se il fattore in esame è presente ad un livello superiore a quello ritenuto normale.

Ogni elemento $x_{i j}\left(x_{i j} \geq 0\right.$ per $i \neq j ; i=1,2, \ldots, n$;

$j=1,2, \ldots, n)$ indica il grado di influenza esercitato dal fattore $x_{i}$ corrispondente alla $i$-esima riga su quello $x_{j}$ corrispondente alla $j$-esima colonna.

Il generico elemento $x_{i j}$ può assumere valore:

- pari a 1 , qualora il fattore $x_{i}$ non presenta alcuna interazione con il fattore $x_{j}$;

- maggiore di 1 , se il fattore $x_{i}$ contribuisce ad esaltare il grado di azione del fattore $x_{j}$;

- compreso fra 0 e 1 , se il fattore $x_{i}$ riduce la potenzialità del grado di azione del fattore $x_{j}$;

- pari a 0 , se il grado di azione del fattore $x_{i}$, ancorché elevato, viene completamente annullato dalla presenza del fattore $x_{j}$.

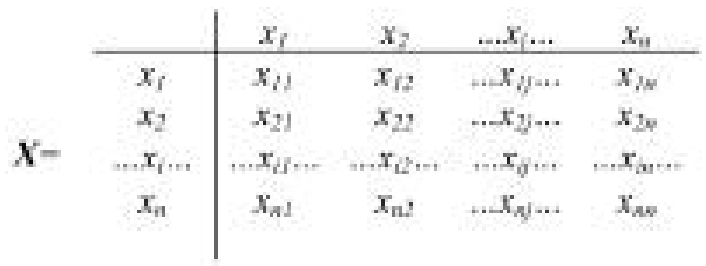

La matrice $\boldsymbol{X}$ può essere orlata con una ulteriore riga (la $n+1$ - esima riga) i cui elementi $v_{j}(j=1,2, \ldots, n)$ sono dati dal prodotto degli $n$ elementi della colonna j-esima, ossia:

$$
v_{j}=x_{j} \cdot x_{2 j} \cdot \ldots \cdot x_{n j}=\prod_{i=1}^{n} x_{i j}(j=1,2, \ldots, n)
$$

Il termine $v_{j}$ rappresenta il grado complessivo d'azione (GCA) esplicitato dal fattore $x_{j}$ come risultato dell'interazione con gli altri $n$ - 1 fattori esaminati.

Per ciascun degli $m$ fabbricati da valutare è, quindi, possibile definire un vettore riga composto dagli $n$ valori del GCA. L'insieme di tali vettori riga definisce la matrice di valutazione $\boldsymbol{M}$ di ordine $m \times n$ :

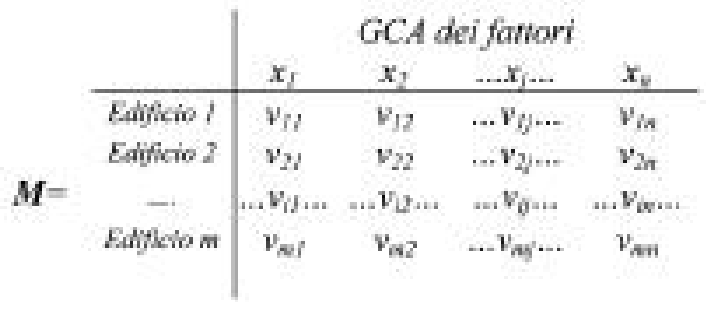

Poiché nell'ambito del campione di edifici le "variabili osservate", e cioè i GCA determinati per ciascun fabbricato, possono essere fra loro correlate e/o possono contenere informazioni ridondanti, a partire dalla matrice $\boldsymbol{M}$, è opportuno definire una o più "variabili artificiali", note anche con il nome di Componenti Principali (CP) [18, 24], che, riducendo il nume- ro delle "variabili osservate", consentono una più agevole interpretazione delle informazioni rilevate. Il numero complessivo di componenti principali è pari al rango della matrice di valutazione $\boldsymbol{M}$. In particolare, la componente principale corrispondente all'autovettore associato al massimo autovalore della matrice di varianza e covarianza calcolata a partire dalla matrice $\boldsymbol{M}$, esplicita i fattori che hanno un peso maggiore nella valutazione del campione di edifici.

Detto $\boldsymbol{p}$ il vettore colonna dei pesi, tale che i suoi elementi $\lambda_{i}$ siano ottenuti elevando al quadrato gli elementi del suddetto autovettore, è possibile pervenire alla classificazione degli $m$ fabbricati mediante la seguente relazione:

$$
\boldsymbol{M} \times \boldsymbol{p}=\boldsymbol{v}^{*}
$$

Il valore assunto dal generico elemento $v_{i}{ }^{*}$, definito grado di apprezzamento totale, consente di stabilire in quale misura l'edificio a cui esso si riferisce può essere adibito alla destinazione funzionale prescelta.

\section{CASO STUdiO}

\section{Case of study}

\subsection{DESCRIZIONE DELL'AREA DI INDAGINE}

\subsection{Description of the surveyed area}

Al fine di verificarne l'efficacia, il metodo proposto è stato applicato ad un'area omogenea della Sicilia sud-orientale che si estende dai Monti Iblei sino alla costa sul Mar Mediterraneo, compresa fra i centri abitati di Ragusa e Santa Croce Camerina.

La prosperità economica della provincia ragusana è determinata soprattutto dalla fiorente attività agricola caratterizzata dall'ortofloricoltura, saldamente insediata lungo la fascia costiera, e dalla zootecnia praticata soprattutto sull'Altopiano. Anche il turismo rappresenta una risorsa economica di rilievo per la provincia iblea, infatti, il flusso turistico registrato nel 2004 presso il complesso delle strutture ricettive ha subito un incremento del $5,3 \%$ e del $7,2 \%$ rispettivamente per arrivi e per presenze turistiche [27]. In particolare, il settore extralberghiero, prevalentemente costituito da campeggi e villaggi turistici ubicati soprattutto lungo la fascia costiera della provincia, presenta negli ultimi dieci anni un trend crescente anche grazie al contributo apportato dall'offerta di alloggi per l'agriturismo.

Gli edifici rurali presenti nell'area considerata sono sostanzialmente riconducibili a tre tipologie: la grande masseria, la piccola masseria e l'abitazione dei piccoli e medi proprietari terrieri [1].

La grande masseria, sviluppatasi soprattutto fra il Seicento e il Settecento, fu espressione del latifondismo. Il proprietario non conduceva direttamente il fondo ma ne cedeva appezzamenti ai contadini in affitto o compartecipazione. Questi ultimi, in genere, non risiedevano permanentemente nella masseria ma, 
giornalmente vi giungevano dai vicini borghi rurali.

La masseria è costituita da un edificio principale, generalmente a pianta quadrata, e da altri fabbricati disposti intorno ad una corte. La casa padronale, spesso costruita su due piani, presenta alcuni elementi architettonici - quali la forma delle aperture esterne, i cornicioni e le grondaie - che richiamano l'architettura cittadina. Le altre costruzioni che sorgono intorno alla corte centrale sono le abitazioni dei salariati e gli annessi rustici, cioè stalle, fienili, granai e locali per la produzione e conservazione dei formaggi.

La piccola masseria, invece, fu espressione delle riforme che, attraverso espropriazioni ed incentivi a favore delle classi contadine, favorirono i frazionamenti fondiari fra le famiglie di fittavoli. Successivamente, però, le difficoltà economiche a cui dovettero far fronte molti piccoli proprietari terrieri comportarono la ricomposizione delle proprietà e la nascita di questo tipo edilizio. Poco diffusa sull' Altopiano Ibleo, ma frequente nell' area di studio, la piccola masseria è caratterizzata dall' assenza della villa padronale e dall'ampliamento della corte esterna.

Contestualmente alla nascita della piccola masseria, si diffusero su tutto il territorio ibleo numerose tipologie edilizie rurali, abitate da contadini le cui attività sociali ed economiche non erano completamente autonome dal grande feudo. Fra queste, il tipo ricorrente è quello della dimora rurale ad una sola elevazione con abitazione e rustico (fienile e stalla) variamente giustapposti (lungo un unico asse, ad "L" oppure a "U") e dislocati spesso attorno ad un piccolo cortile delimitato da muri a secco.

\subsection{QUADRO NORMATIVO NAZIONALE E REGIONALE}

\subsection{National and regional regulation framework}

I costi, generalmente elevati, degli interventi edilizi per il recupero e riuso degli edifici rurali tradizionali non sempre possono essere interamente sostenuti dai proprietari, cosicché la difficoltà di accedere a contributi finanziari costituisce, ancora oggi, l'ostacolo principale all'avvio di progetti e dei relativi lavori.

La Legge 24 dicembre 2003, n. 378 [14] favorisce la salvaguardia e la valorizzazione dell'architettura rurale mediante l'erogazione di contributi ai proprietari fino all'importo massimo del $50 \%$ della spesa da sostenere per gli interventi. La possibilità di accesso al sostegno finanziario è subordinata alla predisposizione da parte delle amministrazioni regionali di appositi programmi triennali che includano criteri di intervento per il risanamento conservativo ed il recupero funzionale degli edifici rurali.

Come previsto dalla suddetta Legge, è stato emanato un apposito Decreto [13] che definisce i criteri tecnico-scientifici per la realizzazione degli interventi edilizi e dà indicazioni per l'individuazione delle diverse tipologie di architettura rurale diffuse sul territorio nazionale. In particolare, la definizione di "architettura rurale" è stata attribuita agli edifici e, più in generale, agli insediamenti realizzati tra il XIII e il
XIX secolo che rappresentano testimonianza significativa "nell'ambito dell'articolazione e della stratificazione storica, antropologica ed urbanistica del territorio, della storia delle popolazioni e delle comunità rurali, delle rispettive economie agricole tradizionali, dell'evoluzione del paesaggio". Dunque, la norma riconosce il legame inscindibile tra l'insediamento rurale, costituito dai fabbricati destinati a residenza e/o ad attività agricole, e lo spazio produttivo.

L'accesso all'aiuto finanziario previsto dalla Legge 24 dicembre 2003, n. 378 è subordinato al rispetto delle norme contenute nel suddetto Decreto e, per gli edifici rurali ubicati in zona sismica, anche all'attuazione di interventi di miglioramento sismico ai sensi delle norme vigenti. Inoltre, ai beni che rivestono interesse storico, artistico o etnoantropologico si applicano le relative disposizioni di tutela previste dal Codice dei Beni Culturali e dei Paesaggi e successive modifiche ed integrazioni [11].

In Sicilia l'Ente regionale attribuisce un ruolo primario al turismo sia per lo sviluppo economico ed occupazionale del territorio sia per la crescita sociale e culturale della collettività. In particolare, la Legge Regionale 15 settembre 2005, n. 10 [15] ha istituito in Sicilia i distretti turistici, contesti omogenei o integrati comprendenti ambiti territoriali appartenenti anche a più province e caratterizzati da offerte qualificate di attrazioni turistiche e/o di beni culturali, ambientali, compresi i prodotti tipici dell'agricoltura e/o dell'artigianato locale. I distretti turistici concorrono allo sviluppo turistico del territorio di competenza attraverso la predisposizione e l'attuazione di specifici progetti finalizzati: alla promozione di strutture ricettive, di servizi e di infrastrutture per il miglioramento dell'offerta turistica; all'individuazione di particolari tipologie di architettura rurale realizzate tra il XII ed il XX secolo (intervallo temporale più ampio di quello fissato dal legislatore nazionale [13]).

La Legge Regionale 15 settembre 2005, n. 10 [15] costituisce un possibile canale di accesso ai contributi finanziari stanziati dalla Legge 24 dicembre 2003, n. 378 [14] poiché prevede l'approvazione di un programma triennale di sviluppo turistico. Pertanto, la possibilità di ottenere contributi economici per il recupero dei fabbricati rurali tradizionali sarà condizionata soprattutto dal loro inserimento all'interno di $d i$ stretti turistici.

Sino ad oggi, però, le linee di intervento in cui si articola la programmazione economica regionale non hanno messo sufficientemente a fuoco i problemi connessi con l'utilizzabilità dell'edilizia rurale tradizionale. In particolare, nelle misure riguardanti "Promozione, adeguamento e sviluppo delle zone rurali" contenute nel Programma Operativo Regionale (P.O.R.) 2000-2006, e finalizzate a consentire la realizzazione di iniziative di turismo rurale e di agriturismo, l'erogazione del finanziamento per il recupero edilizio vincola gli interventi a perseguire il "mantenimento, il rispetto e la tutela delle tipologie e delle forme dei manufatti tradizionali, in un ambito di valorizzazione del- 
le risorse locali, al fine di preservare il paesaggio rurale". Tali obblighi, però, risultano di fatto irrilevanti ai fini della formazione delle graduatorie di ammissibilità al contributo, dato che il valore culturale dell'edificio e la sua compatibilità con l'intervento previsto non contribuiscono alla definizione del punteggio finale con il quale esso è collocato in graduatoria.

Pertanto, al fine di garantire la salvaguardia e la valorizzazione del patrimonio edilizio rurale siciliano e del contesto territoriale in cui esso è ubicato, risulta importante determinare il valore dei due indicatori (cfr. par. 2) relativi alla potenzialità d'uso turistico-ricettiva degli edifici rurali tradizionali oggetto di interventi di recupero e riuso.

\subsection{MATERIALI E METODI}

\subsection{Materials and methods}

Le schede A e B di seguito descritte sono state utilizzate per il rilievo delle informazioni relative a edifici rurali tradizionali parzialmente utilizzati, totalmente inutilizzati o abbandonati, il cui stato di conservazione è tale da non richiedere interventi di recupero edilizio eccessivamente onerosi.

In relazione alla loro rappresentatività nell'ambito delle tipologie precedentemente descritte, sono stati scelti 6 edifici (Fig. 1) per i quali valutare gli indicatori per la determinazione della potenzialità d'uso per fini agrituristici.

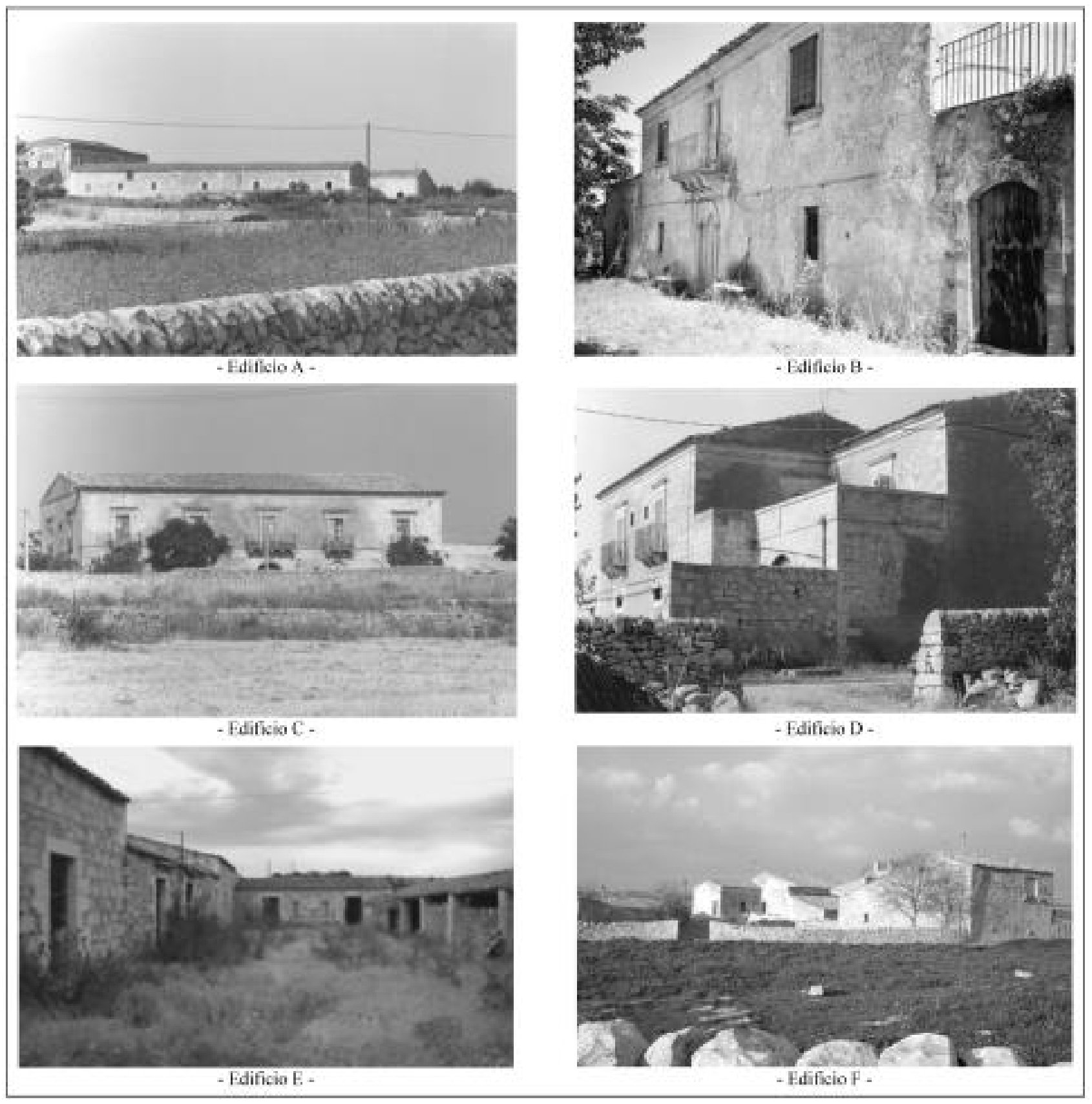

Fig. 1 - Edifici oggetto del caso studio.

Fig. 1 - Buildings of the case of study. 


\subsubsection{Scheda A - Survey card A}

La scheda A consente di rilevare i dati necessari alla valutazione dell' indicatore n. 1: il grado di adattamento della destinazione d'uso agrituristica al contesto locale (Fig. 2). Tale valutazione viene effettuata elaborando le informazioni ottenute sui seguenti otto fattori principali che caratterizzano l'attività agricola dell'azienda e il fondo in cui è ubicato il fabbricato.

a) Fattori che caratterizzano l'attività agricola

- indirizzo produttivo (IP): se presente, può essere vegetale (Ve) e/o zootecnico (Zo). Il primo, in particolare, può essere espletato sotto forma di agricoltura specializzata o consociata; il secondo, invece, può essere intensivo o estensivo;

- modalità di coltivazione del fondo agricolo (MC): può essere distinta in convenzionale $(\mathrm{Co})$, biologica $(\mathrm{Bi})$ o in fase di conversione $(\mathrm{Fc})$ da convenzionale a biologica;

- modalità di trasformazione dei prodotti agricoli $(M T P)$ : se presente, può essere esercitata all'interno dell'edificio o nell' ambito del fondo ad esso annesso o presso altre aziende limitrofe.

b) Fattori che caratterizzano il fondo agricolo

- geometria del fondo (GF): è determinata dalle di- mensioni, dalla pendenza e dalla configurazione del fondo;

- natura del terreno (NT): è definita dalla presenza nel fondo agricolo di roccia affiorante;

- dotazione di servizi (DS): è individuata dal tipo di approvvigionamento idrico ed elettrico e dalla presenza o meno dell'impianto fognario;

- raggiungibilità del fondo agricolo e del fabbricato $(R F F)$ : è definita in relazione al tipo di viabilità (principale o secondaria) e alla distanza del fondo dai più vicini centri abitati.

- possibilità di praticare attività di tempo libero $(A T L)$ : è stabilita in relazione alle attività praticabili nell'ambito del fondo agricolo annesso all'edificio o all'esterno di esso.

\subsubsection{Scheda B - Survey card B}

La scheda B consente di rilevare i dati necessari alla valutazione dell' indicatore n. 2: il grado di adattamento della destinazione d'uso agrituristica ai caratteri morfologici degli edifici rilevati (Fig. 3).

Giacché la valutazione della potenzialità d'uso rappresenta uno studio preliminare sulla possibilità di adibire a specifiche funzioni gli edifici esistenti, per la compilazione della scheda non è necessario compiere

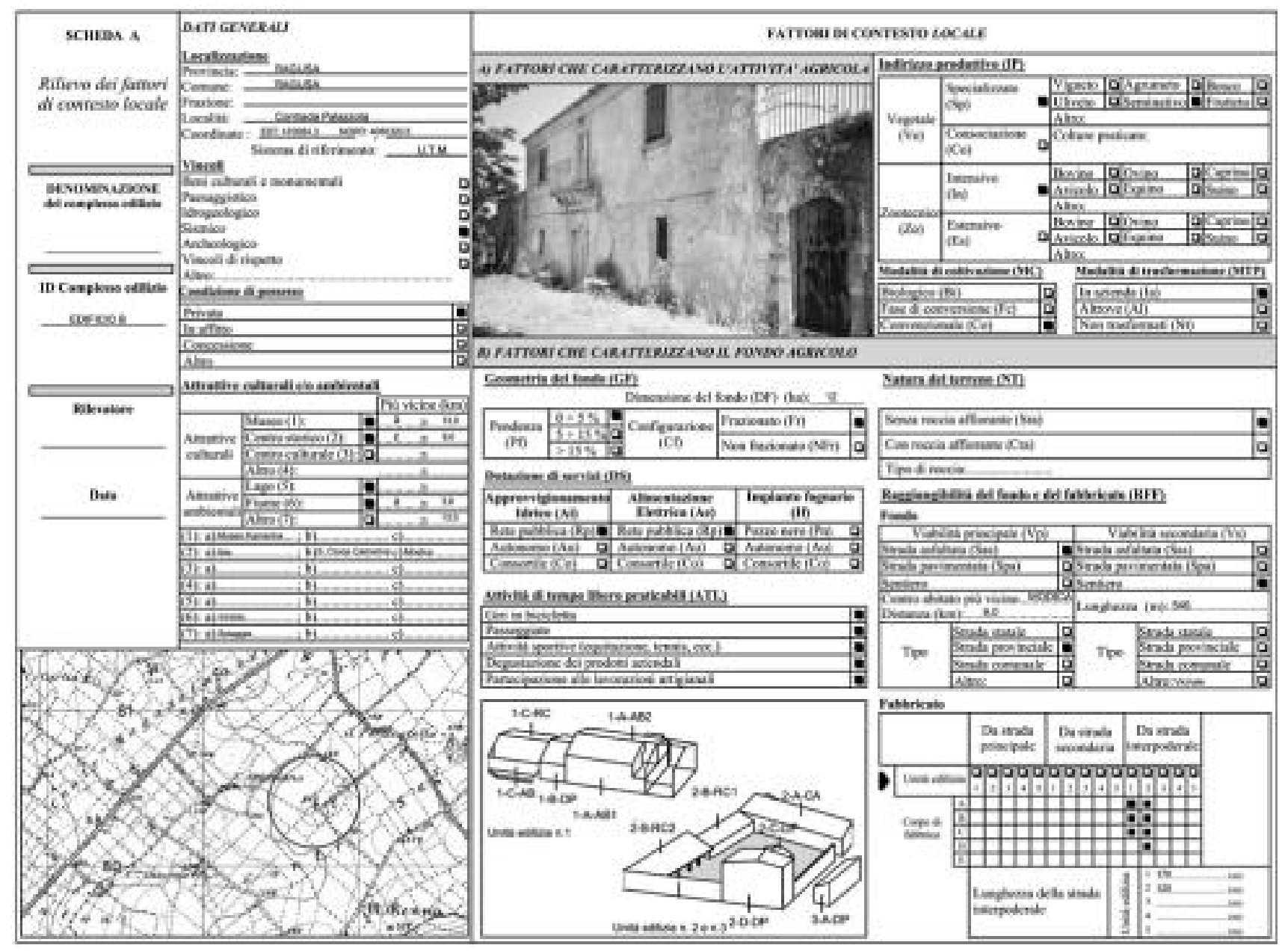

Fig. 2 - SchedaA.

Fig. 2 - Survey card A. 
rilievi metrici di dettaglio; infatti, al fine di formulare un giudizio di compatibilità fra le esigenze dalla nuova destinazione d'uso e la morfologia dell'edificio, si ritiene sufficiente rilevare soltanto alcuni dati geometrici indispensabili per definire le dimensioni di massima dell'organismo edilizio (superfici e altezze interne delle unità funzionali).

Nella prima parte della scheda è possibile inserire, in forma grafica e/o alfanumerica, i dati relativi alla collocazione planimetrica delle unità edilizie che compongono il complesso rurale e alla loro scomposizione in corpi di fabbrica.

Nella seconda parte della scheda possono essere annotati, attenendosi alle indicazioni grafiche ed alle note illustrative appositamente inserite, i dati relativi ai caratteri morfologici e allo stato di conservazione del fabbricato.

3.3.3 Indicazioni grafiche contenute nella scheda B - Graphical aids included in the survey card B

a) Tipi di spazio

Nella classe ST1 sono stati raggruppati gli spazi singoli. Tali spazi, in particolare, possono essere di modeste dimensioni qualora siano adibiti ad abitazioni temporanee di pastori e/o agricoltori, a ricovero di animali, a deposito di attrezzi agricoli, mentre possono assumere dimensioni rilevanti se utilizzati per l'immagazzinamento dei prodotti agricoli (granai, cantine, ecc.), per la stabulazione di un numero elevato di animali (stalle per l'allevamento delle bovine da latte, scuderie), per le attività religiose della comunità rurale, ecc.

Alla classe ST2 appartengono gli spazi aggregati di piccole e medie dimensioni. Si tratta, generalmente, di ambiti funzionali adibiti ad abitazione padronale stagionale o a dimora permanente che, seppur degradati, presentano finiture interne migliori rispetto agli spazi di tipo ST1.

Nella classe ST3 sono stati raggruppati gli ambiti caratterizzati da un'articolata morfologia degli spazi interni determinata, generalmente, dalle esigenze di attività specializzate connesse con la lavorazione dei prodotti agricoli (palmenti, oleifici, mulini, ecc.).

b) Tipi di chiusura orizzontale

Le chiusure orizzontali di tipo $\mathrm{SC} 1$ sono quelle realizzate con volte vere o con volte finte. Le prime hanno funzione portante, possono essere realizzate a conci o a getto e si riscontrano, generalmente, nelle case padronali come elementi di chiusura orizzontale tra due livelli sovrapposti. Le seconde, invece, realiz-

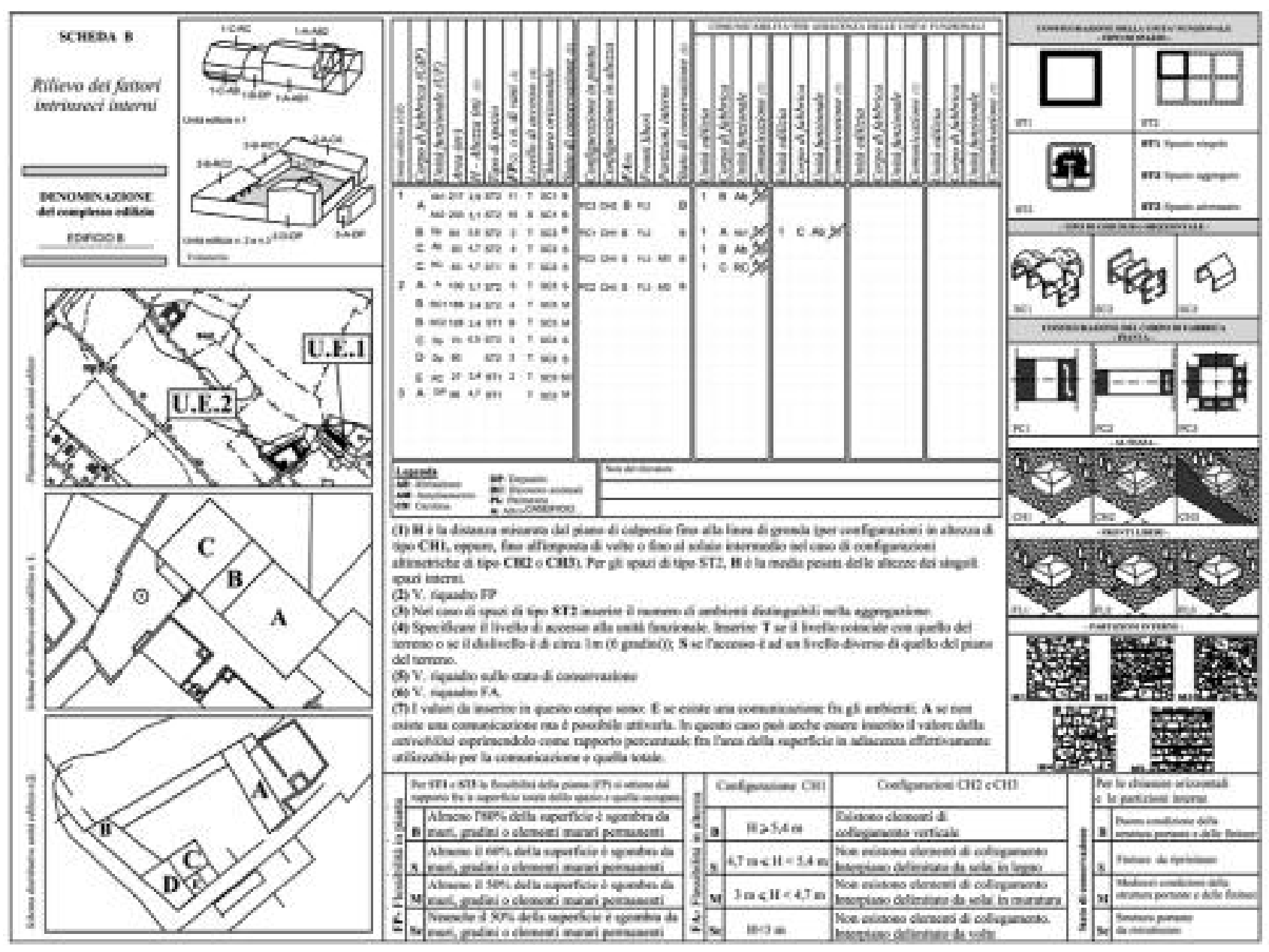

Fig. 3 - Scheda $B$.

Fig. 3 - Survey card B. 
zate in canne e gesso, fungono da controsoffitto ed assumono, pertanto, una funzione estetica. Nelle unità funzionali con volte vere gli interventi di ristrutturazione sono particolarmente onerosi e delicati. In particolare, è generalmente difficoltoso, se non addirittura impossibile, eliminare una o più partizioni interne per unificare ambienti adiacenti ed adattarli alla nuova destinazione funzionale. D'altra parte, in presenza di volte vere o finte, la suddivisone in ambienti di minore superficie richiede particolari accorgimenti di tipo estetico. Infatti, la qualità geometrica dello spazio chiuso da un sistema voltato viene compromessa dalle nuove partizioni verticali. Anche la possibilità di mettere in collegamento unità funzionali poste a livelli diversi (flessibilità dello spazio in altezza) può essere notevolmente limitata dalla presenza delle volte. La realizzazione di scale e/o ascensori, infatti, oltre a ledere la funzione estetica del sistema voltato può pregiudicarne in modo rilevante anche la funzione statica a causa delle necessarie bucature da realizzare.

Le chiusure orizzontali di tipo SC2 sono rappresentate dai solai piani. Essi consistono in un tavolato orizzontale poggiato su travi di legno. Sopra il tavolato, in relazione alla destinazione funzionale degli ambienti, può essere realizzata una pavimentazione costituita da mattonelle in cotto. Questo tipo di chiusura orizzontale si riscontra, generalmente, negli edifici adibiti ad abitazione contadina e in quelli utilizzati per il ricovero degli animali. In questo ultimo caso, il tavolato privo di pavimentazione veniva utilizzato per il deposito di paglia e/o fieno. Anche nelle realizzazioni più complesse il solaio orizzontale garantisce una certa flessibilità dello spazio in pianta e in altezza. Negli interventi di ristrutturazione, infatti, esso può essere forato per la realizzazione di elementi di collegamento verticale mentre eventuali partizioni interne necessarie alla nuova funzione non compromettono la qualità estetica dello spazio chiuso superiormente con un solaio piano.

Il tipo di chiusura SC3 rappresenta la classica copertura a falde inclinate. Essa, essendo generalmente priva di controsoffitto, lascia in evidenza l'orditura strutturale della copertura. Negli interventi di ristrutturazione la chiusura a tetto offre il beneficio di potere ampliare la cubatura degli spazi interni al fine di assicurare i requisiti minimi richiesti dalle norme igienico sanitarie vigenti. In relazione alla destinazione d'uso degli ambienti, però, questo tipo di chiusura può richiedere oneri economici rilevanti per la realizzazione della controsoffittatura.

c) Configurazione in pianta

Nell'abaco sono state rappresentate graficamente le configurazioni planimetriche che possono influenzare le soluzioni distributive di una nuova destinazione funzionale.

Appartengono alla classe PC1 i corpi di fabbrica che hanno forma prevalentemente rettangolare con larghezza del fronte minore della profondità.

Viceversa, appartengono alla classe PC2 i corpi di fabbrica a pianta rettangolare con larghezza del fronte maggiore della profondità. Infine, nella classe PC3 rientrano i corpi di fabbrica nei quali la larghezza del fronte è paragonabile alla profondità.

d) Configurazione in altezza

La configurazione altimetrica si riferisce al numero di livelli dei corpi di fabbrica e alla loro superficie di sovrapposizione.

Appartengono al tipo $\mathrm{CH} 1$ gli edifici ad unica elevazione ovvero quelli che, pur presentando un volume complessivo rapportabile a due o più elevazioni fuori terra, non presentano chiusure orizzontali intermedie.

I corpi di fabbrica con configurazione in altezza di tipo $\mathrm{CH} 2$ hanno volume complessivo risultante dalla sovrapposizione di due o più livelli fuori terra. La possibilità di utilizzare i piani superiori per destinazioni funzionali che determinano un incremento significativo dei carichi, è subordinata ad una verifica statica della struttura nelle nuove condizioni di esercizio.

Appartengono al tipo $\mathrm{CH} 3$ i corpi di fabbrica a più elevazioni sfalsate. Tale condizione, in relazione all'andamento altimetrico del terreno, può risultare vantaggiosa per la possibilità di realizzare accessi esterni autonomi ai diversi piani, serviti da viabilità esterna. L'ampiezza della zona di sovrapposizione, però, condiziona la possibilità di realizzare efficacemente i collegamenti verticali interni e gli spazi distributivi e di connessione.

e) Fronti liberi

Il numero e la disposizione dei fronti liberi dell'unità edilizia (FL1, FL2, FL3) condizionano i livelli di illuminazione e ventilazione naturali esistenti e/o realizzabili e gli aspetti distributivi in relazione alla possibilità di realizzare nuove aperture di accesso.

f) Tipi di muratura

In relazione agli eventuali interventi di recupero da realizzare, un altro fattore rilevante nella descrizione complessiva del corpo di fabbrica è rappresentato dal tipo di muratura che costituisce le partizioni interne. È opportuno che l'abaco inserito nella scheda di rilievo B contenga i tipi di muratura ricorrenti nei corpi di fabbrica in relazione alle tradizioni costruttive dell'area d'indagine.

\section{ANALISI DEI DATI E DISCUSSIONE DEI RISULTATI}

\section{Analysis of data and discussion}

\subsection{INDICATORE N. 1}

\subsection{Indicator $n .1$}

Le informazioni contenute nelle schede di rilievo A sono state elaborate impiegando il modello multicriteriale precedentemente descritto (cfr. par. 2.2). Tali elaborazioni hanno consentito la valutazione dell'indicatore n. 1, cioè del grado di adattamento della destinazione d'uso agrituristica al contesto locale dei fabbricati oggetto di studio, mediante la determinazione del grado di apprezzamento totale, calcolato con la formula 4. 


\begin{tabular}{c|cccccccc} 
& IP & GF & NT & RFF & DS & MC & MTP & ATL \\
\hline IP & 1,80 & 1,00 & 1,00 & 1,00 & 1,00 & 1,00 & 1,00 & 1,00 \\
GF & 1,50 & 1,50 & 1,00 & 1,00 & 1,00 & 1,00 & 1,00 & 1,50 \\
NT & 1,00 & 1,00 & 1,00 & 1,00 & 1,00 & 1,00 & 1,00 & 1,00 \\
RFF & 1,00 & 1,00 & 1,00 & 1,00 & 1,00 & 1,00 & 1,00 & 1,00 \\
DS & 1,00 & 1,00 & 1,00 & 1,00 & 1,00 & 1,00 & 1,50 & 1,00 \\
MC & 1,00 & 1,00 & 1,00 & 1,00 & 1,00 & 1,50 & 1,00 & 1,00 \\
MTP & 1,00 & 1,00 & 1,00 & 1,00 & 1,00 & 1,00 & 1,50 & 1,00 \\
ATL & 1,00 & 1,00 & 1,00 & 1,00 & 1,00 & 1,00 & 1,00 & 1,70 \\
\hline GCA & 2,70 & 1,50 & 1,00 & 1,00 & 1,00 & 1,50 & 2,25 & 2,55
\end{tabular}

Fig. 4 - Matrice di interazione $\boldsymbol{X}$ riferita ad uno dei fabbricati considerati nello studio e relativo grado complessivo di azione (GCA) dei fattori.

Fig. 4 - Compiled matrix $\mathbf{X}$ for one of the studied buildings and degree of global action (DGA) of the factors.

Per ciascun fabbricato è stata costruita la matrice $X$ di ordine 8 , le cui 8 righe ed 8 colonne si riferiscono agli 8 fattori da analizzare (Fig. 4). I valori dei termini della diagonale sono stati assegnati facendo riferimento ai criteri di valutazione riportati in Fig. 5.

Per assicurare oggettività al processo di valutazione dell'indicatore considerato i valori da attribuire ai criteri sono stati definitivi tenendo in considerazione le priorità funzionali della destinazione d'uso dettate sia dalla normativa vigente, che regolamenta l'attività agrituristica, sia dalle norme di buona progettazione.

I valori al di fuori della diagonale, sono stati inseriti tenendo conto dell'interazione tra $\mathrm{i}$ fattori secondo quanto previsto nel modello. Ad esempio, la valutazione del fattore indirizzo produttivo (IP) è influenzata dal tipo di servizi disponibili in azienda (DS) (idrico, elettrico, ecc.) e, soprattutto se l'azienda ha indirizzo produttivo prevalentemente vegetale, dalla natura del terreno (NT) e dalla geometria del fondo (GF). Invece, il fattore modalità di trasformazione dei prodotti agricoli (MTP) è fortemente influenzato oltre che dai servizi disponibili (DS), dalla raggiungibilità del fondo agricolo e/o del fabbricato (RFF), soprattutto se i prodotti agricoli necessitano del conferimento ad altre aziende per la loro trasformazione.

Impiegando la relazione (2), ciascuna matrice $\boldsymbol{X}$ è stata orlata con una ulteriore riga i cui elementi $v$ $(j=1,2, \ldots, n)$ sono dati dal prodotto degli 8 elementi della colonna j-esima. Le righe così ottenute per ciascun fabbricato esprimono i gradi complessivi d'azione (GCA) esercitati dai fattori e formano la matrice di valutazione riportata in Fig. 6, utilizzata per il calcolo dell'indicatore $\mathrm{n}$. 1. Per determinare il vettore dei pesi $\boldsymbol{p}$ (Fig. 7) è stata applicata l'Analisi delle Componenti Principali (cfr. par. 2.2) alla matrice riportata in Fig. 6. Il modesto peso assunto dai fattori GF, NT, RFF, DS, MC (Fig. 7) non implica la loro scarsa importanza nell' ambito del processo valutativo ma indica che essi assumono valori poco variabili nell'ambito del campione esaminato.

Infine, la Tab. 1 contiene il grado di adattamento della destinazione d'uso agrituristica al contesto lo- cale (indicatore n. 1) dei 6 fabbricati analizzati nel caso studio, determinato applicando la relazione 4.

I valori dell'indicatore n. 1 riportati in Tab. 1 mostrano l'elevato grado di adattamento della destinazione d'uso agrituristica al contesto locale dei fabbricati, superando mediamente dell' $83 \%$ circa il valore unitario considerato come livello normale nel modello di valutazione adottato (cfr. par. 1.2).

I risultati riportati in Tab. 1 sono condizionati prevalentemente dai valori attribuiti nelle relative matrici di interazione $\boldsymbol{X}$ ai fattori IP, MTP e ATL, nonché dal loro grado complessivo d'azione determinato dall'interazione con tutti gli altri fattori (GF, NT, RFF, DS ed MC) mediante la relazione (2). Inoltre, quattro dei fabbricati analizzati hanno ottenuto valori dell'indicatore $\mathrm{n} .1$ più elevati per effetto del grado complessivo d'azione del fattore IP (Fig. 6). Infatti, la presenza di allevamenti zootecnici estensivi, talvolta associati a produzioni vegetali, ha consentito di attribuire al fattore IP punteggi maggiori rispetto al livello unitario ritenuto normale (Fig. 5).

Analoghe considerazioni possono essere svolte per il fattore MTP. Infatti, la presenza di attività di trasformazione dei prodotti agricoli con tecniche tradizionali ha consentito di attribuire a tale fattore punteggi maggiori rispetto al livello unitario ritenuto normale e, anche in questo caso, il calcolo del grado complessivo d'azione per tale fattore MTP ha determinato un incremento nel $50 \%$ dei fabbricati oggetto di studio (Fig. 6).

La possibilità offerta ai turisti di praticare attività di intrattenimento, quali la partecipazione alle lavorazioni artigianali, la degustazione di prodotti tipici, le visite guidate presso centri culturali, le escursioni nelle riserve naturali, ha permesso di assegnare punteggi elevati anche al fattore ATL, che è risultato inferiore al livello ritenuto normale soltanto per uno degli edifici considerati nel caso studio (Fig. 6).

\subsection{INDICATORE N. 2}

\subsection{Indicator $n .2$}

Come riportato in studi condotti precedentemente [4], per la valutazione del grado di adattamento della destinazione d'uso agrituristica ai caratteri morfologici (indicatore n. 2) dei fabbricati oggetto di studio sono stati analizzati $\mathrm{i}$ dati contenuti nella scheda B, nonché i requisiti richiesti dalla destinazione d'uso considerata $[2,12,16,17]$, pervenendo alla definizione dei seguenti fattori che ne influenzano la valutazione [4]:

Pzn: numero delle possibili soluzioni distributive per la realizzazione della zona notte;

Pzr: numero delle possibili soluzioni distributive per la realizzazione della zona ristorazione;

CRzn: capacità ricettiva della zona notte, determinata dal numero di utenti contemporaneamente ospitabili;

CRzr: capacità ricettiva della zona ristorazione, determinata dal numero di coperti contemporaneamente o fruibili; 
CRITERI DI VALUTAZIONE DEL GRADO DI AZIONE DEI FATTORI CHE CARATTERIZZANO L'ATTIVITA' AGRICOLA

Per la valuatacione di questo fattore viene privilegiata la rilevante importanza degli allevamenti zootecnici nell'ambito delle attivita agricole praticate nell' Altopiano Ibleo, soprattutto per la valorizzare delle produzioni locali ed dei caratteri distietivi del paesaggio agrario. A tal proposito, l'allevamento estensivo o semieshensivo è stato preferito a quello intensivo, praticato provalentemente nelle modeme stalke per bovini (da ingrasso o da latte), poiché il pascolamento dei capi, oltre a caratterizzare e valorizzare il paesaggio, consente un'alimentazione pǜ naturale per gli animali con coeseguente miglioramento della qualità della produzione di latte e dei suoi derivati (sopratutio ricotta e formaggio) che raporesentano i prodotti tiposi della zona. Invece, nella valutazicee di questo fatto-

IP

$\left(\mathrm{x}_{11}\right)$ re per le aziende agricole ad indirizzo prevalentemenie vegetale, viene preferita la pratica dell' agricoltura consociata poiché le specie coltivase, oltere ad imegrarsi con il paesaggio sircostanle, offroeso una produzione piú varia da utilizzare nell'esereizio đell'antività agrituristica.

\begin{tabular}{|c|c|c|c|c|c|c|c|}
\hline & $\begin{array}{l}\text { Senza indirizos prodat- } \\
\text { tivo (IP) ma potenzial- } \\
\text { mente attivabile }\end{array}$ & \multicolumn{2}{|c|}{$\begin{array}{l}{[\mathrm{P}-\{\mathrm{Ve}\} \text { oppure }} \\
{[\mathrm{P}=\{\mathrm{Z} O\}}\end{array}$} & \multicolumn{2}{|c|}{$\begin{array}{l}\text { Ve- }\{\mathrm{Co}\} \text { oppure } \\
\mathrm{Z}_{0}=\{\mathrm{Es}\}\end{array}$} & $\begin{array}{l}\mathrm{IP}=\{\mathrm{Ve}\}\}_{e} \\
\mathrm{IP}=\{\mathrm{Z} 0\}\end{array}$ & 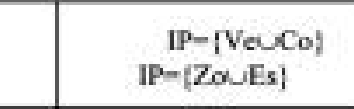 \\
\hline & 0.5 & \multicolumn{2}{|c|}{1,0} & \multicolumn{2}{|c|}{1,5} & 1,8 & 2.0 \\
\hline \multirow{3}{*}{$\begin{array}{l}\mathrm{MC} \\
\left(\mathrm{x}_{\mathrm{ses}}\right)\end{array}$} & \multicolumn{7}{|c|}{$\begin{array}{l}\text { La modalita di coltivazione poò essere biologica, convenzionale o in fase di conversioese da tradizionale a biologica. Si rítiene che } \\
\text { un'azienda ehe pratica la coltivazione biologica possa richiamare un maggior numero di turisti. }\end{array}$} \\
\hline & \multicolumn{3}{|c|}{ 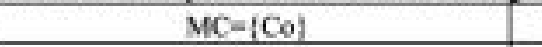 } & \multicolumn{2}{|c|}{$\mathrm{MC}-\{\mathrm{Fe}\}$} & \multicolumn{2}{|c|}{$\mathrm{MC}=[\mathrm{Bi} i]$} \\
\hline & \multicolumn{3}{|c|}{\begin{tabular}{r|r}
0,5 & \\
\end{tabular}} & \multicolumn{2}{|c|}{1,0} & \multicolumn{2}{|r|}{1.5} \\
\hline \multirow{3}{*}{$\begin{array}{l}\operatorname{MTP} \\
(\mathrm{x} \rightarrow)\end{array}$} & \multicolumn{7}{|c|}{$\begin{array}{l}\text { La pratica dei procedimenti tradizionali per la trasformazione dei prodotti agricoli puó offrire un qualificante beneficio alle consuni- } \\
\text { tà locali poiché garantisce il trasferimento alle generazioni future di metodi e tecniche di trasformazione che appartengoeo alla tra } \\
\text { dizione. Pertanto, nella valutaziose vengono privilegiale le aziende che trasformano i prodotti agricoli con tecriche tradizionali. }\end{array}$} \\
\hline & \multicolumn{3}{|c|}{$\mathrm{MTP}-\{\mathrm{N} t\}$} & \multicolumn{2}{|c|}{$\mathrm{MTP}-\{\mathrm{A} \mid\}$} & \multicolumn{2}{|r|}{$\mathrm{MC}-\{1 \mathrm{a}\}$} \\
\hline & \multicolumn{3}{|c|}{\begin{tabular}{r|l}
0 & \\
\end{tabular}} & \multicolumn{2}{|c|}{1,0} & \multicolumn{2}{|c|}{1,5} \\
\hline \multicolumn{8}{|c|}{ CRITERI DI VALUTAZIONE DEL GRADO DI AZIONE DEI FATTORI CHE CARATTERIZZANO IL FONDO AGRICOLO } \\
\hline \multirow[t]{3}{*}{$\begin{array}{c}\text { GF } \\
\left(\mathrm{x}_{n z}\right)\end{array}$} & \multicolumn{7}{|c|}{ 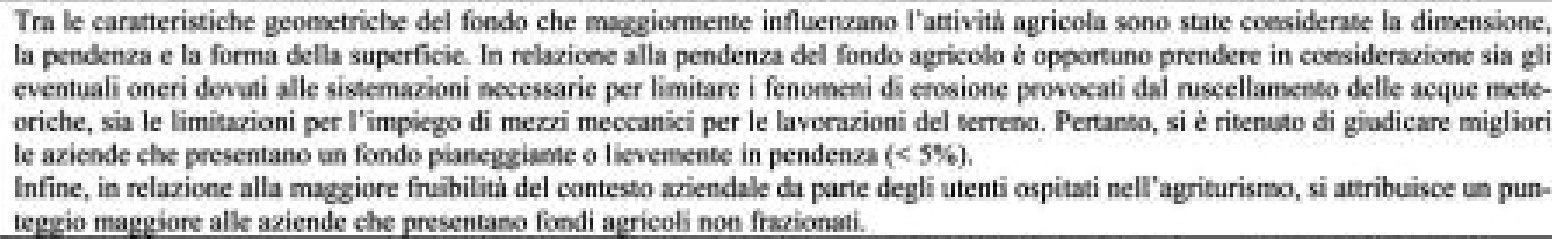 } \\
\hline & $\mathrm{DF}>2 \mathrm{~h}, \mathrm{PF}<15 \%$ & & \multicolumn{2}{|c|}{$\mathrm{DF}>2 \mathrm{hn}, \mathrm{PF}<15 \%, \mathrm{CF}=[\mathrm{Fr}\}$} & \multicolumn{2}{|c|}{$\mathrm{DF}>2 \mathrm{~h}_{2}, \mathrm{PF}<15 \%, \mathrm{CF}=[\mathrm{NFr}]$} & In tuati gli altri casi \\
\hline & 1.0 & & \multicolumn{2}{|c|}{1,2} & \multicolumn{2}{|c|}{1.5} & 0,5 \\
\hline \multirow{3}{*}{$\begin{array}{l}\text { NT } \\
(\mathrm{x} n)\end{array}$} & \multicolumn{7}{|c|}{$\begin{array}{l}\text { Poiché la presenza di roccia affionante paò condizionare la possibelití di svolgere alcune attivita di tempo libero, è opportuno pren- } \\
\text { dere in esame gli oneri economici eonnessi con la realizzazione degli spiamamenti éo dezli scavi necessari per mighiorare la frnibeli- } \\
\text { tà del fondo. }\end{array}$} \\
\hline & \multicolumn{4}{|c|}{$\mathrm{NT}-[\mathrm{Sra}]$} & \multicolumn{3}{|c|}{$\mathrm{NT}-\{\mathrm{Cra}\}$} \\
\hline & & & & & & & \\
\hline RFF & $\begin{array}{l}\text { Poiché l'accessibilità del } \\
\text { cui e possibile raggiunge } \\
\text { viaria (principale e secon } \\
\text { se si percorrono strade pe }\end{array}$ & & $\begin{array}{l}\text { uò influenrare } \\
\text { turiseno, si ritic } \\
\text { alla distanza d. } \\
\text { te piutlosto ch }\end{array}$ & $\begin{array}{l}\text { ibilita di } \\
\text { ortune va } \\
\text { ipali cent: } \\
\text { in terra b }\end{array}$ & $\begin{array}{l}\text { ambio dei } \\
\text { tare la qua } \\
\text { abitati. Ino } \\
\text { tuta. }\end{array}$ & $\begin{array}{l}\text { thi con i me } \\
\text { gealizrazio } \\
\text { sso al fond }\end{array}$ & $\begin{array}{l}\text { titi locali nonché la facilith con } \\
\text { del fondo in relazione alla rete } \\
\text { n automobile risulta preferibile }\end{array}$ \\
\hline & $\begin{array}{r}\text { RFF-rVpus: } \\
\text { [Vsspas }\end{array}$ & & & $\begin{array}{r}\mathrm{RFF}=\{\mathrm{V} \\
\text { IVs }\end{array}$ & $\begin{array}{l}\text { USas; e } \\
\text { Sas; }\end{array}$ & & In tuati gli altri casí \\
\hline & 1,0 & & & & 5 & & 0.5 \\
\hline $\begin{array}{c}\text { DS } \\
\left(\mathrm{x}_{95}\right)\end{array}$ & $\begin{array}{l}\text { Giacche Tenergia elettri } \\
\text { ''imtratienimesto dei turis } \\
\text { relarione al tipo di appro } \\
\text { doesta di approwigionan } \\
\text { te allacciamento consorti. }\end{array}$ & & $\begin{array}{l}\text { qua potabile } \\
\text { nda, epport } \\
\text { mento dispon } \\
\text { co aunonomo } \\
\text { ete pubblica. }\end{array}$ & $\begin{array}{l}\text { mportanti } \\
\text { siderare: } \\
\text { tonomo, } \\
\text { sia prefe }\end{array}$ & $\begin{array}{l}\text { per lo sva } \\
\text { oneri ecor } \\
\text { onsortile, re } \\
\text { bile rispet: }\end{array}$ & $\begin{array}{l}\text { Lelle attivi } \\
\text { ntualment } \\
\text { a). Inoltre. } \\
\text { lotene đi af }\end{array}$ & $\begin{array}{l}\text { gricole ed indispensabili per } \\
\text { vessari per gli allaceiamenti in } \\
\text { itiene che un'azienda agricola } \\
\text { vigivasmesto idrico median- }\end{array}$ \\
\hline & Senza servizi & & $D S-\{A]\}$ & AE) & $\begin{aligned} \mathrm{DS}=1 \\
\mathrm{Al}\end{aligned}$ & eon & $D S-\{A \mid \cup A a\} e\{A E \cup R p\}$ \\
\hline & 0 & & & & & & 1,5 \\
\hline & $\begin{array}{l}\text { La possibilità di svolger } \\
\text { fine di assicurare una piü }\end{array}$ & & $\begin{array}{l}\text { di tempo liber } \\
\text { rmanenza dei }\end{array}$ & $\begin{array}{l}\text { equitazic } \\
\text { a azienda }\end{array}$ & giri in & sseggiata & assume un ruolo rilevante al \\
\hline $\begin{array}{l}\text { ATL } \\
\left(\mathrm{x}_{\text {sed }}\right)\end{array}$ & $\begin{array}{l}\text { Impossibilità di pratican } \\
\text { vità di tempo libero }\end{array}$ & & $\begin{array}{l}\text { Se è possibil } \\
\text { un tipo di at } \\
\text { li }\end{array}$ & Ire solo & Secp & rasticare pià & ana attivith di tempo libero \\
\hline & $\theta$ & & & & & $\begin{array}{l}1,0+ \\
\text { di attività o }\end{array}$ & $\begin{array}{l}0,1) \\
\text { empo libero praticabili. }\end{array}$ \\
\hline
\end{tabular}

Fig. 5 - Criteri di valutazione del grado di azione dei fattori che caratterizzano l'attività agricola e il fondo (il significato degli acronimi è contenuto nella scheda $A$ ).

Fig. 5 - Assessment criteria of the degree of action of the factors related to the agricultural activity and to the farm (the meaning of the acronyms is contained in the survey card A). 


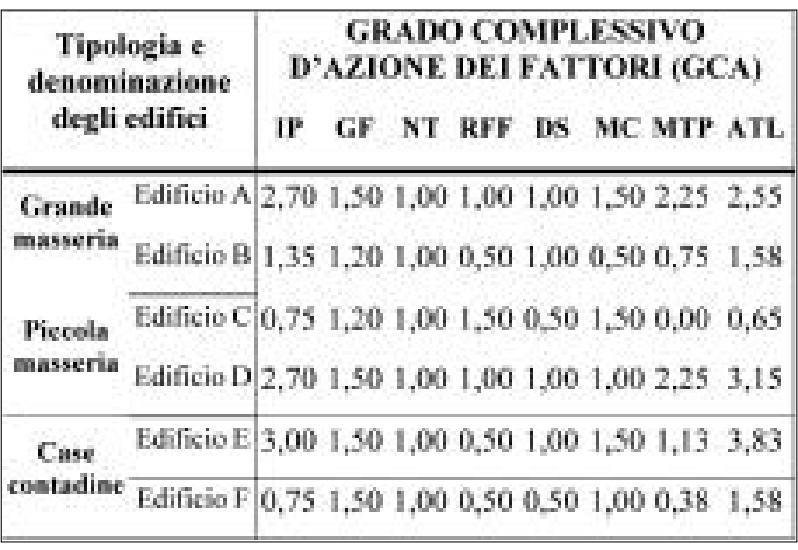

Fig. 6 - Matrice di valutazione M per il calcolo dell'indicatore n. 1 .

Fig. 6 - Matrix $\boldsymbol{M}$ for the assessment of the indicator n. 1.

\begin{tabular}{|cc|c|c|c|c|c|c|c|}
\hline \multicolumn{1}{c|}{ IP } & GF & NT & RFF & DS & MC & MTP & ATL \\
\hline \multirow{2}{*}{ pesi } & $\lambda . \mathrm{fc}_{7}$ & $\lambda \mathrm{fc}_{2}$ & $\lambda \mathrm{fc}_{3}$ & $\lambda . \mathrm{fc}_{4}$ & $\lambda \mathrm{fc}_{5}$ & $\lambda . \mathrm{fc}_{6}$ & $\lambda \mathrm{fc}_{7}$ & $\lambda \mathrm{fc}_{8}$ \\
\cline { 2 - 8 } & 0,345 & 0,004 & 0,000 & 0,003 & 0,015 & 0,003 & 0,222 & 0,409 \\
\hline
\end{tabular}

Fig. 7 - Vettore dei pesi $\boldsymbol{p}$.

Fig. 7 - Weight vector $\boldsymbol{p}$

\begin{tabular}{|l|c|}
\hline Edifici & IVDicatore N. 1 \\
\hline Edificio A & 2,501 \\
\hline Edificio B & 1,298 \\
\hline Edificio C & 0,545 \\
\hline Edificio D & 2,745 \\
\hline Edificio E & 2,875 \\
\hline Edificio F & 1,004 \\
\hline
\end{tabular}

TABella 1 - Indicatore n. 1 - Grado di adattamento della destinazione d'uso agrituristica al contesto locale dei fabbricati del caso studio.

TABLE 1 - Indicator n. 1 - Degree of adaptability of the tourist use to the local context, calculated for the buildings of the case of study.

DZF: disponibilità di altre zone funzionali (oltre la zona notte e la zona ristorazione) fruibili dagli ospiti; SMzn: superficie mediamente disponibile per utente, calcolata dividendo la superficie complessiva degli ambienti da adibire a zona notte per il numero di ospiti; ST: tipo di spazio utilizzato per la realizzazione della zona per la ristorazione;

TCO: tipo di chiusura orizzontale dell'ambiente adibito alla zona notte ed alla zona ristorazione;

MTsa: media degli scarti tollerabili delle superfici degli ambiti funzionali costituenti la zona ristorazione rispetto alle superfici richieste;

MTaa: media degli scarti tollerabili delle altezze degli ambiti funzionali costituenti la zona ristorazione rispetto alle altezze richieste;

Per ciascuno dei fabbricati analizzati sono state elaborate apposite schede di sintesi (Fig. 8) contenenti i dati da impiegare per valutare $\mathrm{i}$ fattori sopra individuati. I criteri impiegati per tale valutazione sono stati definiti in studi precedenti [4] tenendo in considerazione il sistema di norme che regolamenta l'esercizio dell'attività agrituristica in Sicilia [16, 17].

Applicando il modello già utilizzato per la determinazione dell'indicatore $\mathrm{n} .1$ (cfr. par. 5.1), in Fig. 9 è riportata la matrice di valutazione $\boldsymbol{M}$ e in Fig. 10 il vettore dei pesi $\boldsymbol{p}$ calcolato mediante l'ACP. Anche in questo caso, come per l'indicatore n. 1, il modesto peso attribuito ai fattori PZn, SMzn, TCO, Pzr, CRzr, ST, MTsaa e MTaa (Fig. 10) non implica la loro scarsa importanza nell' ambito del processo valutativo ma indica che essi assumono valori poco variabili all'interno del campione esaminato.

Infine, la Tab. 2 contiene il grado di adattamento della destinazione d'uso agrituristica ai caratteri morfologici (indicatore n. 2) dei 6 fabbricati analizzati nel caso studio, determinato applicando la relazione 4 .

I valori dell'indicatore n. 2 riportati in Tab. 2 mostrano una modesta suscettività turistico-ricettiva degli edifici oggetto di studio. Infatti, il grado di adattamento della destinazione agrituristica ai caratteri morfologici di 4 dei 6 fabbricati analizzati non raggiunge il livello unitario considerato normale dal modello di valutazione, risultando al di sotto di tale soglia mediamente del $73 \%$ circa. Tale risultato è condizionato prevalentemente dai valori attribuiti nella matrice di interazione $X$ ai fattori CRzn e DZF, nonché dal loro grado complessivo d'azione determinato dall'interazione con tutti gli altri fattori (PZn, SMzn, TCO, Pzr, CRzr, ST, MTsaa e MTaa) mediante la relazione.

In particolare, il valore attribuito al fattore CRzn risulta fortemente condizionato da un lato dal criterio di valutazione adottato [4], che prevede l'attribuzione di un valore inferiore al livello unitario ritenuto normale qualora non sia possibile realizzare un numero di posti letto superiore a 27 (cioè pari alla metà del numero massimo consentito dalle norme vigenti in Sicilia [16, 17]), dall'altro dall'interazione con gli altri fattori introdotti per la valutazione dell'indicatore n. 2 (SMzn, TCO, ST, MTsaa e MTaa).

Infatti, allorché la distribuzione degli ambienti interni degli edifici non consente di raggiungere gli standard edilizi imposti dalle norme e dai regolamenti vi-

\begin{tabular}{|l|c|}
\hline Emfic & INDICATORE N. 2 \\
\hline Edificio A & 0,317 \\
\hline Edificio B & 2,471 \\
\hline Edificio C & 0,178 \\
\hline Edificio D & 1,399 \\
\hline Edificio E & 0,525 \\
\hline Edificio F & 0,064 \\
\hline
\end{tabular}

TABella 2 - Grado di adattamento della destinazione d'uso turistica ai caratteri morfologici dei fabbricati oggetto del caso studio.

TABLE 2 - Degree of adaptability of the tourist use to the morphological features of the buildings of the case of study. 
genti, il valore iniziale del fattore CRzn, fissato in relazione al numero di posti letto realizzabili, a causa della sua interazione con i fattori SMzn, MTsaa ed MTaa subisce un decremento. Inoltre, un ulteriore decremento del valore del fattore CRzn è determinato dalla sua interazione con i fattori TCO ed ST che ten- gono in considerazione gli interventi edilizi necessari per la realizzazione degli ambienti adibiti a zona notte, qualora gli spazi a disposizione non ne siano idonei.

I valori assunti dal fattore DZF (Fig. 9) indicano la scarsa possibilità di realizzare alcuni ambienti necessari per l'accoglienza degli ospiti (salette di soggior-

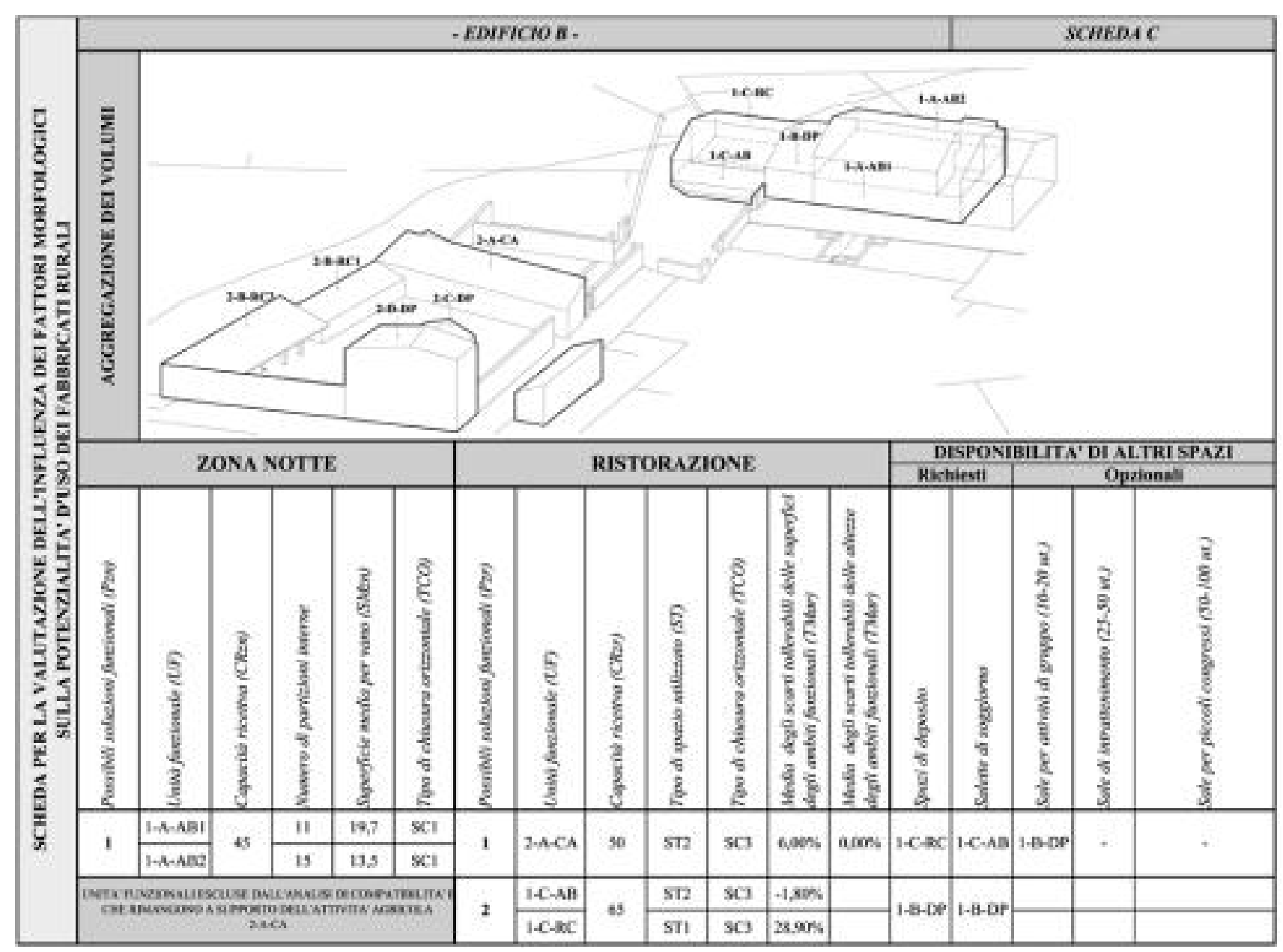

Fig. 8 - Scheda di sintesi contenente i dati da impiegare per la valutazione dei fattori relativi all'indicatore $n$. 2 .

Fig. 8 - Compiled card containing data needed for the assessment of the indicator n. 2 .

\begin{tabular}{|c|c|c|c|c|c|c|c|c|c|c|c|}
\hline \multirow{2}{*}{\multicolumn{2}{|c|}{ Tipologia edilizia }} & \multicolumn{10}{|c|}{ GRADO COMPLESSIVO D'AZIONE DEI FATTORI (GCA) } \\
\hline & & Pzn & CRzn & SMzn & TCO & $\mathrm{Pzr}$ & CRur & ST & Mitsa & Mtaa & DZF \\
\hline \multirow{2}{*}{$\begin{array}{l}\text { Grande } \\
\text { masseria }\end{array}$} & Edificio A & 1,00 & 0,21 & 0.50 & 0,15 & 1,00 & 0,70 & 0,30 & 1,00 & 1,00 & 0.50 \\
\hline & Edificio B & 1,00 & 2,88 & 1,15 & 0,28 & 1,50 & 0,65 & 0,24 & 0,80 & 1,00 & 1,65 \\
\hline \multirow{2}{*}{$\begin{array}{l}\text { Piccela } \\
\text { masseria }\end{array}$} & Edificio C & 1,00 & 0,19 & 0,50 & 0,05 & 1,00 & 0,24 & 0,02 & 1,00 & 1,00 & 0,00 \\
\hline & Edificio D & 1,30 & 1,50 & 0,13 & 0,03 & 1,00 & 0,22 & 0,12 & 0,80 & 1,00 & 1,30 \\
\hline \multirow{2}{*}{$\begin{array}{l}\text { Case } \\
\text { contadine }\end{array}$} & Edificios E & 1,00 & 0,51 & 0,50 & 0,15 & 1,00 & 0.65 & 0.15 & 1,00 & 1,00 & 0.50 \\
\hline & Edificio F & 1,00 & 0,05 & 0.56 & 0,10 & 1,000 & 0.13 & 0.15 & 0,60 & 1,00 & 0.00 \\
\hline
\end{tabular}

Fig. 9 - Matrice di valutazione M per il calcolo dell'indicatore n. 2.

Fig. 9 - Matrix $\mathbf{M}$ for the assessment of the indicator n. 2.

\begin{tabular}{|c|c|c|c|c|c|c|c|c|c|c|}
\hline \multirow{3}{*}{ Pesi } & $P_{z A}$ & CRom & SMzn & TCO & $P_{z z r}$ & CRer & ST & Mitsa & Mtaa & DZFF \\
\hline & $2 \mathrm{fm}_{4}$ & $\lambda \mathrm{fm}_{2}$ & $\operatorname{sim}_{3}$ & $\lambda \mathrm{fm}_{4}$ & $\mathrm{xfm}_{\mathrm{s}}$ & $\lambda \mathrm{fm}_{6}$ & $2 \mathrm{fm}$ & $\lambda \mathrm{fm}_{\mathrm{x}}$ & 2.fm4 & $2 . \mathrm{fm}_{H}$ \\
\hline & 0,001 & 0,694 & 0,017 & 0,002 & 0,018 & 0,005 & 0,001 & 0,000 & 0,018 & 0,245 \\
\hline
\end{tabular}

Fig. 10 - Vettore dei pesi $\boldsymbol{p}$.

Fig. 10 - Weight vector $\boldsymbol{p}$. 
no) e per lo sgombero e/o pulizia dell' agriturismo (depositi). Soltanto tre dei fabbricati analizzati consentono la realizzazione dei suddetti ambienti e, di conseguenza, il relativo fattore DZF possiede un valore maggiore del livello ritenuto normale.

\section{CONCLUSIONI}

\section{Conclusions}

Il metodo descritto nel presente studio ha consentito la determinazione di due indicatori per la valutazione della potenzialità d'uso turistico-ricettiva di edifici rurali tradizionali: il grado di adattamento della destinazione d'uso degli edifici al contesto locale (indicatore n. 1); il grado di adattamento della destinazione d'uso ai caratteri morfologici degli edifici (indicatore n. 2).

Poiché la scelta di tali indicatori scaturisce dai contenuti del Decreto Ministeriale 6 ottobre 2005 [13] il metodo illustrato può essere applicato per valutare la potenzialità d'uso di edifici rurali tradizionali ubicati in altre realtà nazionali, diverse da quelle considerate in questo studio.

Al fine di rendere quanto più esauriente ed obiettiva la valutazione degli indicatori, il metodo proposto ha previsto l'impiego di un modello basato sull'analisi multicriteriale in cui i criteri di valutazione dei fattori individuati sono stati stabiliti facendo riferimento alle priorità funzionali della destinazione d'uso nonché alle norme di buona progettazione.

Si ritiene che, nell'ambito di processi di valutazione finalizzati al finanziamento di progetti di recupero e riuso di edifici rurali tradizionali, la conoscenza dei suddetti indicatori può contribuire alla tutela e alla salvaguardia del patrimonio architettonico rurale tradizionale sancite dalla Legge 24 dicembre 2003, n. 378 [14], poiché consente l'individuazione di edifici compatibili con la destinazione d'uso prescelta (agriturismo, turismo rurale, Bed and Breakfast, ecc.).

L'applicazione del metodo proposto in un'area omogenea della Sicilia orientale, la cui valenza turistica può comportare l'interesse di classificare $\mathrm{i}$ fabbricati rurali in essa ubicati in relazione alla loro potenzialità d'uso agrituristica, ha evidenziato che tale destinazione d'uso seppure presenti un grado di adattamento al contesto locale (indicatore n. 1) complessivamente al di sopra del livello ritenuto normale, non sempre è compatibile con i caratteri morfologici degli edifici (indicatore n. 2). Infatti, l'analisi condotta ha evidenziato che soltanto due fabbricati fra i sei analizzati si prestano bene ad essere riutilizzati per lo svolgimento di attività agrituristiche (Edificio $\mathrm{B}$ ed Edificio D). Tale risultato evidenzia l'importanza che il metodo proposto può assumere nell' ambito dei programmi di recupero e riuso dell'edilizia rurale tradizionale. Esso, infatti, considerato un insieme di edifici rurali tradizionali ricadenti in un' area omogenea, attraverso una serie di elaborazioni che tendono a minimizzare le valutazioni soggettive, consente di indivi- duare gli edifici che presentano un più elevato grado di suscettività al riuso per attività turistico-ricettive. Tale metodo, pertanto, può integrare i criteri di valutazione adottati dalle Autorità competenti, per la stesura delle graduatorie di approvazione delle istanze di contributo finanziario per la realizzazione di attività turistico-ricettive.

\section{BIBLIOGRAFIA}

\section{References}

[1] Aa.Vv. (1973). La casa rurale nella Sicilia Orientale. C.N.R., "Ricerche sulle dimore rurali in Italia", Olschki editore, Firenze.

[2] AA.Vv. (1996). Manuale di progettazione edilizia Fondamenti, strumenti, norme - Tipologie e criteri dimensionali. 1, Hoepli, Milano.

[3] Cascone G., Di Fazio S., Pennisi P. (1993). Tendenze in atto e proposte progettuali per il riuso dei fabbricati rurali. Atti del V Congresso Nazionale AIGR su "Il ruolo dell'Ingegneria per l'Agricoltura del 2000", Maratea, 177-185.

[4] Cascone G., Porto S.M.C., La Pergola P. (2005). Il rilievo delle informazioni per la classificazione funzionale degli edifici rurali tradizionali. Atti del VIII Convegno Nazionale dell'AIIA su 'L'ingegneria agraria per lo sviluppo sostenibile dell' area mediterranea", 27-30 giugno 2005, Catania.

[5] Di FAZIO S., FAILla A. (1994). The reuse of traditional farm buildings in the context of a changing agriculture. Proceedings of the CIGR XII World Congress on Agricultural Engineering, Milano, 1, 661670.

[6] Di FAZIO S., FicherA C.R. (2001). Architettura rurale e potenzialità di sviluppo del turismo nella provincia di Reggio Calabria - Uno studio di fattibilità nell'area del Basso Tirreno Reggino. Laruffa Editore, Reggio Calabria.

[7] Della SpINa L. (2004). Procedure di valutazione della qualità abitativa. Gangemi Editore, Roma.

[8] Failla A., Cascone G., Porto S.M.C. (2003). Approccio metodologico per il recupero sostenibile dell'edilizia rurale tradizionale. R.U.R.A.L.I.A. "La tutela dell'architettura rurale nell'evoluzione del sistema produttivo", Società Editrice Fiorentina, Firenze, 131-144.

[9] FICHERA C.R. (1997). Tutela e valorizzazione degli edifici rurali storici e tradizionali. Genio Rurale, n. 1, 63-68.

[10] Fusco GIRARD L. (1987). Risorse architettoniche $e$ culturali, valutazioni e strategie di conservazione. Franco Angeli, Milano.

[11] Gazzetta Ufficiale Repubblica Italiana (2006). Disposizioni correttive ed integrative al decreto legislativo 22 gennaio 2004, n. 42, in relazione ai beni culturali, Decreto Legislativo 24 marzo 2006 n. 156, G.U.R.I. del 27 aprile 2006 n. 97, Roma.

[12] Gazzetta Ufficiale Repubblica Italiana (2006). Disciplina dell'agriturismo, Legge 20 febbraio 2006 n. 96, G.U.R.I. n. 63 del 16 marzo 2006, Roma.

[13] GazzetTA UfFiciale RepubBlicA ItAliana (2005). Individuazione delle diverse tipologie di architettura rurale presenti sul territorio nazionale e definizione 
dei criteri tecnico-scientifici per la realizzazione degli interventi, ai sensi della Legge 24 dicembre 2003, $n$. 378, recante disposizioni per la tutela e la valorizzazione della architettura rurale, Decreto Ministeriale 6 ottobre 2005, G.U.R.I. n. 238 del 12 ottobre 2005, Roma.

[14] GazZetta Ufficiale Repubblica Italiana (2004). Disposizioni per la tutela e la valorizzazione dell'architettura rurale, Legge 24 dicembre 2003 n. 378, G.U.R.I. n. 13 del 17 gennaio 2004, Roma.

[15] Gazzetta Ufficiale Regione Siciliana (2005). Norme per lo sviluppo turistico della Sicilia e norme finanziarie urgenti, Legge Regionale 15 settembre 2005 n. 10, G.U.R.S. n. 39 del 16 settembre 2005, Palermo.

[16] Gazzetta Ufficiale Regione Siciliana (2004). Disposizioni in materia di agriturismo, Assessorato dell'Agricoltura e delle Foreste, D.A. 28 maggio 2004 n.568, G.U.R.S. n. 2618 giugno 2004, Palermo.

[17] Gazzetta Ufficiale Regione Siciliana (1994), Norme sull'agriturismo, Legge Regionale 9 giugno 1994, n. 25, G.U.R.S. n.30 del 14 giugno 1994, Palermo.

[18] Hardle W., Simar L. (2003). Applied Multivariate Statistical Analysis. Springer Verlag, Berlin-Heidelberg, New York.

[19] Kalman H. (1980). Evaluation des Bâtiments Historique. Environnement Canada, Service des parcs, Ottawa.

[20] Margani L., Salemi A. (1988). Materiali e tecniche costruttive della tradizione siciliana. Tre studi su Catania. Documenti - I.D.A.U (Istituto Dipartimentale di Architettura e Urbanistica), n. 16 Idonea Giovanni Litografo, Catania.

[21] Mennella V.G.G., Menconi M.E. (2006). Old buildings in the landscape. Rivista di Ingegneria Agraria, 3, 57-64.

[22] Mennella V.G.G. (1997). Qualità dell'ambiente $e$ sviluppo delle aree rurali. Genio Rurale, 1, 75-78.

[23] PorTo S.M.C. (2003). Definizione di un metodo per la valutazione della potenzialità d'uso degli edifici rurali tradizionali. Tesi di Dottorato di ricerca in "Costruzioni agricole e assetto del territorio", Università degli Studi di Catania, Dipartimento di Ingegneria Agraria, Catania.

[24] Rencher A.C. (2002). Methods of multivariate analysis. $2^{\text {nd }}$ Edition. Wiley Interscience. John Wiley $\&$ Sons, New York.

[25] Rizzo F. (1993). Economia del patrimonio architettonico ambientale. Franco Angeli, Milano.
[26] Ruda G. (1998). Rural buildings and environment. Landscape and urban planning 41, 93-97.

[27] SISTAN - Sistema Statistico Nazionale (2007). Ragusa in cifre. Edizione 2006. La Grafica, Modica (RG).

[28] VAN DeR VAART J.H.P. (2005). Towards a new rural landscape: consequences of non-agricultural re-use of redundant farm buildings in Friesland. Landscape and urban planning 70, 143-152.

[29] Zavadskas E.K., Antucheviciene J. (2007). Multiple criteria evaluation of rural building's regeneration alternatives. Building and Environment 42, 436-451.

\section{Parole chiave:}

valutazione, edifici rurali tradizionali, agriturismo

\section{SUMMARY}

\section{INDICATORS FOR ASSESSING TOURIST POTEN- TIAL USE OF TRADITIONAL RURAL BUILDINGS}

To assess tourist potential use of traditional rural buildings two indices are determined. Indicator $n .1$ regards the degree of adaptability of the tourist use to the territorial context; indicator $n$. 2 regards the degree of adaptability of the tourist use to the morphological features of the buildings. The method which aims to assess the above-mentioned indices is divided into two phases. The first phase regards the knowledge of the traditional rural buildings. Survey cards A and B allow to get information needed for the assessment of the factors which influence the two indices. The second phase provides the application of a multicriteria model which allows the assessment of the two indices.

The method has been applied to six traditional rural buildings sited in a homogeneous area in south eastern Sicily. The results show that although the degree of adaptability of the tourist use to the territorial context is high for almost all the considered buildings (indicator n. 1), only two buildings have a high degree of adaptability of the tourist use to the morphological features (indicator n. 2).

Key words:

assessment, traditional rural buildings, farm holidays. 